\title{
OPEN THORACOTOMY AND DECORTICATION FOR CHRONIC EMPYEMA (FIBROTHORAX)
}

P. B. Nichkaode, S. Dasgupta, Rahul Zamad, Nilesh Tulaskar, Abhay Chaudhary

1. Associate Professor. Department of Surgery, NKP Salve Institute of Medical Sciences, Nagpur

2. Dean \& Professor. Department of Surgery, NKP Salve Institute of Medical Sciences, Nagpur

3. Junior Resident. Department of Surgery, NKP Salve Institute of Medical Sciences, Nagpur

4. Senior Resident. Department of Surgery, NKP Salve Institute of Medical Sciences, Nagpur

5. Lecturer. Department of Surgery, NKP Salve Institute of Medical Sciences, Nagpur

\section{CORRESPONDING AUTHOR:}

Dr. Rahul C. Zamad,

27, Congress Nagar,

Tapsya bhavan ,

Nagpur- 440012.

E-mail: zamad.rahul@gmail.com

ABSTRACT: OBJECTIVES AND AIMS: Traditionally, chronic empyema has been treated by thoracotomy and decortication. Some recent reports have claimed similar clinical results for video thoracoscopy, but with less morbidity and mortality than open procedures. Our experience with thoracotomy and decortication in patients in fibrothorax stage is reviewed so that the results of this surgical procedure can be adequately evaluated. MATERIALS AND METHODS: From September 2007 to march 2012, 126 patients diagnosed with empyema thoracis were treated in NKP Salve Institute of medical sciences. Diagnosis of chronic empyema was based on the duration of signs and symptoms before definitive treatment and imaging findings, such as constriction of the lungs and the thoracic cage. Fifty six patients fulfilled the criteria for chronic empyema and underwent open thoracotomy and decortication. RESULTS AND STATISTICS: Thirty nine patients (69.6\%) were males and the mean age of study group was 31.4 years. Etiology was synpneumonic effusion in 49 patients (87.5\%), subpleural malignancy in 3 patients, traumatic haemothorax in 2 patients and ruptured hydatid cyst in 2 patients . The mean duration of symptoms and signs before definitive treatment averaged 42 days. All patients had chronic empyema, as confirmed by imaging studies and operative findings. There were 3 patients (5.4\%) with complications and one mortality. The postoperative length of stay averaged 11.2 days. There were no recurrences of empyema. Complete expansion of lung was noticed in 53 patients at end of 3 months on follow up. CONCLUSION: Treatment for patients with chronic empyema thoracis with fibrothorax can be achieved with Open thoracotomy and decortication with low complication rates and low mortality with good results.

KEYWORDS: Pleural empyema, Chronic empyema, Decortication, Video-thoracoscopy.

INTRODUCTION: The treatment of thoracic empyema has evolved since its inception by Hippocrates. ${ }^{1,2}$ Improvements of equipment and thoracoscopic techniques in the 1990s helped for the expansion of applications of video-thoracoscopy. One indication for video-thoracoscopy is the treatment of empyema. There is general agreement that empyemas in the fibrinopurulent stage should be treated thoracoscopically; however, treatment at the chronic stage remains controversial. One point of contention is that thoracotomy and decortication involve increased morbidity and mortality. ${ }^{6,7}$ Importantly, there are also reports which indicate that morbidity 
and mortality are low when using this procedure.13, 14 To that end, several thoracic surgeons consider thoracotomy and decortication to be the best treatment for chronic empyema. ${ }^{8-12}$

We have reviewed our experience with thoracotomy and decortication in the management of chronic empyema, focusing on morbidity, mortality, postoperative management and functional results.

MATERIAL AND METHODS: The hospital where study has been carried out is a tertiary care hospital and majority of patients attending the hospital are from rural area

This is a descriptive, retrospective and observational study. Records of patients who were treated for empyema in the hospital between September 2007 and March 2012 were reviewed. The information obtained from the records included sex, age, origin, habits, morbidity, etiology of empyema, blood tests, imaging study findings, results of smears, cultures of pleural fluids, biochemical parameters of empyema, signs/symptoms and their duration until definitive surgery, procedures performed before definitive surgery, length of operation, duration of chest intubation, postoperative days in the hospital, complications, mortality and follow-up.

Diagnosis of empyema was confirmed by one of the following criteria: (1) drainage of grossly purulent pleural fluid, (2) pleural fluid culture or Gram stain showing bacteria or (3) biochemical parameters of empyema $(\mathrm{pH}<7.2$, lactate dehydrogenase level $>1,000 \mathrm{IU} / \mathrm{L}$, glucose level $<40 \mathrm{mg} / \mathrm{dl}$.). Chronic empyema was defined in accordance with the American Thoracic Society staging system, ${ }^{15}$ where stage III empyema corresponds to chronic empyema or the organizing stage. This diagnosis was corroborated by illness durations of more than 15 days before definitive treatment as well as supportive imaging findings, such as constriction of the chest cavity.

The surgical protocol consisted of double lumen intubation and placement of an epidural catheter for analgesia. The surgical approach was orientated by imaging study findings using limited incision or postero-lateral thoracotomies; the latter were used for large or loculated empyemas. Surgery included evacuation of all purulent material and formal decortication with the aim of obtaining the largest possible pulmonary expansion. In most cases, decortication of parietal pleura was partial and performed in accordance with the need for complete decortication of the visceral pleura. Single chest tube was inserted at the end of the operation. No pleural irrigation or fibrin sealant was used in any case ${ }^{3,4,8}$. (FIGURE 1)

Patients were extubated in the operating room and sent to the ward for postoperative management. Patients were managed in the intensive care unit only when mechanical ventilation or vasopressors were required. Aggressive respiratory therapy was performed. Chest tubes were removed when there was no air leakage and when the drainage was less than $50 \mathrm{ml}$ per day for two consecutive days. Follow-up took place in the outpatient clinic and when needed, patients and were contacted telephonically.

RESULTS AND STATISTICS: One hundred twenty six patients diagnosed with empyema were treated over a period of 4.5 years. Of these, 58 satisfied the criteria for chronic empyema. Two patients were excluded from analysis as they were managed with placement of an empyema tube as these patients did not undergo thoracotomy and decortication due to high surgical risk. The remaining 68 cases were treated with tube thoracostomy, video-thoracoscopy or open thoracotomy (either limited or postero-lateral) and decortication, and will not be discussed. 
Thus, 56 patients were managed with the previously described surgical protocol and form the basis of this study. Thirty nine patients (69.6\%) were male and seventeen $(30.4 \%)$ were female. The average age of the patients was 31.4 years (range 14 to 69 years). The cause of chronic empyema was synpneumonic effusion in 49 (87.5\%) subpleural malignancy in 3 patients (5.3\%) ,trauma in 2 patients (3.5\%)and ruptured hydatid cyst in 2 patients(3.5\%) . of these 34 cases were reported as non specific inflammation, 19 were tuberculous and three were malignant .on preoperative pulmonary function test 37 patients had restrictive and 19 had obstructive lung diseases.

The most frequent habits included 31 (55.3\%) patients with alcoholism, 27 (48.2\%) smokers. Co-morbidities consisted of 4 (7.1\%) patients with Diabetes mellitus type II, two patients each with bronchial asthma and high blood pressure and AIDS was reported in one case $(1.8 \%)$.

Symptoms included coughing in 49 (87.5\%) patients, chest pain and fever in 38 patients $(67.8 \%)$ and fever $(>/=38.5)$ in 34 patients $(60.7 \%)$. Dyspnea was found in $41(73.2 \%)$ patients. Thirty nine (69.6\%) patients had leukocytosis. The average white blood cell count was 12,566 per $\mathrm{mm}^{3}$ (range 3,900 to 26,400 per $\mathrm{mm}^{3}$ ), and twenty-six (46.4\%) patients were anemic. The average $\mathrm{Hb}$ was $10.4 \mathrm{~g} / \mathrm{dl}$ (range 8.6 to $13.5 \mathrm{~g} / \mathrm{dl}$ ) and $9.4 \mathrm{~g} / \mathrm{dl}$ (range 8.0 to $11.6 \mathrm{~g} / \mathrm{dl}$ ) for men and women, respectively.

All patients were treated with antibiotics for a variable period of time before being admitted to our unit. Twenty four patients underwent chest tube insertions before definitive surgery was undertaken. Pleural fluid was routinely cultured and bacterial growth was found in 20 (35.7\%) samples. Among the assessed biochemical parameters, lactic dehydrogenase was consistently elevated, with an average value of 5162.4 UI/L (range 1,124 - 9,320 UI/L). The average time prior to definitive surgery was 43 days (range 15 to 90 days). The chest tube was removed after an average of 5 days (range 2 to 28 days). Fifty three patients did not have complications. Two patients developed prolonged air leakage (bronchopleural fistula) that was managed with a Heimlich valve and In one patient, it was necessary to leave an empyema tube for 4 weeks due to purulent drainage and a residual small empyema cavity. There was no mortality or recurrence of empyemas. The average postoperative stay was 11.2 days (range 5 to 42 days). The patient who remained in the hospital for 42 days after thoracotomy and decortication was septic with empyema cavity. There was no recurrence of empyema or mortality.

DISCUSSION: It is generally accepted that pleural empyema should be treated early to avoid complications, extensive operations and lengthy hospital stays. ${ }^{6,16}$ Unfortunately, there are some patients for whom early treatment is not possible and in whom chronic empyema will develop, mainly due to delayed diagnosis or delayed referral. At this stage, the standard treatment is open thoracotomy and decortication. Historically, other surgical procedures have been practiced, including open window thoracostomy, rib resection, thoracoplasty, Claggett's procedure and percutaneous drainage guided by ultrasound or CT. ${ }^{17,18}$ With the increasing popularity of minimally invasive techniques, video-thoracoscopy has been proposed for the treatment of chronic empyemas.3,4,7,19 There are reports in which open procedures have shown higher morbidity and mortality.6, 7 It is for these reasons that we decided to analyze our experience with open thoracotomy and decortication in the management of chronic empyemas. In western world most cases of empyema are diagnosed easily in first two stages. So the best possible treatment is video assisted surgery. In Indian scenario usually patients present late and 
this entity is chronic and in form of fibrothorax. There are lots of technical difficulties in VATS for injuring the underlying lung parenchyma in this patient. So open thoracotomy and decortication is the best treatment option in these patients. Many patients in early stages are treated only with tube thoracostomy and are then neglected for any thoracoscopic intervention for early empyema.

In our cases, pleural fluid cultures showed bacterial growth in $35.7 \%$ of patients, which is comparable to several previous reports.5,8,9,10 Additionally, lactic dehydrogenase was consistently elevated with an average value of $5162.4 \mathrm{UI} / \mathrm{L}$. Soriano et al. ${ }^{16}$ established a direct relationship between higher levels of lactic dehydrogenase and longer disease duration. Imaging studies consisted of chest roentgenograms, ultrasound and chest CT. These studies demonstrated a thick pleural cortex and constriction of the thoracic cage. Chest CT was particularly useful for showing the pleural cortex, loculations, size of the empyema and evidence of constriction of the thoracic cage. There are no specific indicators to confirm the chronicity of the empyemas; ${ }^{20}$ however, in this series, the presence of the pleural cortex and constriction of the thoracic cage coupled with evolution times of more than 15 days was indicative of chronic empyema in all cases. During surgery, reduction of the intercostal spaces and the fibrous pleural cortex were evident; these are features of chronic empyema. In addition to showing the characteristics of the empyemas, chest CT allows the surgeon to locate and determine the best surgical approach, either limited or postero-lateral thoracotomy. We have routinely performed chest CT before surgery for all patients diagnosed with empyema at our institute. (figure 2) In the present work, it is important to note that chest intubation time averaged five days, and postoperative stay averaged 11.2 days. Only three patients presented complications and there was no mortality. These results show shorter duration of chest intubation, fewer postoperative days and less or similar complications rate and mortality as compared to patients treated with video-thoracoscopy. ${ }^{4,7}$

The definitive results of the surgical procedure were assessed using the scheme proposed by Melloni et al.,14 which takes into account elimination of symptoms, normalization of the white blood cell count, removal of pleural drains, return to pre-illness physical activities, and roentgenography. The results of all patients in this study were classified as "very good". Symptoms resolved, white blood cell counts became normal, no patients required a new pleural drain (one patient had a chest tube converted to an empyema tube for 4 weeks), and patients returned to their regular activities by postoperative week 4 . Roentgenography did not show residual pleural effusion, and pulmonary expansion was complete or slightly diminished. Spirometry for evaluation of functional results was not used on a regular basis, as there was no points of comparison since many patients were too ill to establish a pre-surgery baseline. Additionally, spirometry during illness would not reflect the real spirometric values of the patient. ${ }^{7}$

This study had several limitations; the number of cases was relatively small, although all patients were operated on by a single surgeon using the same approach and technique. The range of number of days from symptom manifestation to definitive surgery might be considered low, but these patients were classified using the staging system of the American Thoracic Society. ${ }^{15}$ Some reports ${ }^{19}$ have classified patients as having phase III empyema without indicating the number of days before definitive treatment which is not the case in this series and also the number of days before surgery was undertaken was less in other studies. It is not always possible to recognize the exact beginning of the disease process due to the indolent presentation of the infection in many patients. 
Empyema treatment is included in the category $3 \mathrm{~b}$ level of evidence-based medicine and expert opinions. ${ }^{21}$ In our study, open thoracotomy and decortication was found to be an excellent surgical procedure with low morbidity and mortality. Functional results were also excellent, as all patients returned to the normal activities that they performed before surgery. Open thoracotomy and decortication is the standard treatment for chronic empyema, but there have been no prospective, randomized studies comparing open thoracotomy and decortication with video-thoracoscopy.

CONCLUSION: Open thoracotomy and decortication meant for those patients who have chronic empyema with fibrothorax were there is technical difficulty in VATS for fear of underlying lung parenchymal injury can be achieved with low morbidity and mortality. Long-term functional results are especially promising. More studies comparing open method and minimal access method are necessary to define with certainty which patients would benefit from open thoracotomy and which ones from video-thoracoscopy.

\section{REFERENCES:}

1. Yim APC. Paradigm shift in empyema management. Chest. 1999;115:611-12.

2. Somers J, Faber LP. Historical developments in the management of empyema. Chest Surg Clin North Am. 1996;6:403-18.

3. Waller DA, Rengarajan A. Thoracoscopic decortication: A role for video-assisted surgery in chronic postpneumonic pleural empyema. Ann Thorac Surg. 2001;71:1813-16.

4. Cheng YJ, Wu HH, Chou SH, Kao EL. Video-assisted thoracoscopic surgery in the treatment of chronic empyema thoracics. Surg Today.2002;32:19-25.

5. Roberts JR. Minimally invasive surgery in the treatment of empyema: intraoperative decision making. Ann Thorac Surg. 2003;76:225-30.

6. Luh SP, Chou MC, Wang LS, Chen JY, Tsai TP. Video-assisted thoracoscopic surgery in the treatment of complicated parapneumonic effusions or empyemas. Chest. 2005;127:1427-32.

7. Chan DT, Sihoe AD, Chan S, Tsang DS, Fang B, Lee TW, et al. Surgical treatment of empyema thoracis: Is video-assisted thoracic surgery "Better" than thoracotomy ? Ann Thorac Surg. 2007;84:225-31.

8. Wurnig PN, Wittmer V, Pridun NS, Hollaus PH. Video-assisted thoracic surgery for pleural empyema. Ann Thorac Surg. 2006;81:309-13.

9. Renner H, Gabor S, Pinter H, Maier A, Friehs G, Smolle-Juettner FM. Is aggressive surgery in pleural empyema justified? Eur J Cardiothorac Surg. 1998;14:117-22.

10. Lardinois D, Gock M, Pezzetta E, Buchli C, Rousson V, Furrer M, et al. Delayed referral and gram-negative organisms increase the conversion thoracotomy rate in patients undergoing video-assisted thoracoscopic surgery for empyema. Ann Thorac Surg. 2005;79:1851-5.

11. Cassina PC, Hauser M, Hillejan L, Greschuchna D, Stamatis G. Video-assisted thoracoscopy in the treatment of pleural empyema: stage-based management and outcome. J Thorac Cardiovasc Surg. 1999;117:234-8.

12. Petrov D, Dzhambazov V, Petkova P, Boianova L, Minchev TS, Goranov E, et al. Surgical treatment of chronic pleural empyema-seven year's experience. Khirrurgiia. 2004;60:25-29. 
13. LeMense GP, Strange C, Sahn SA. Empyema thoracis. Therapeutic management and outcome. Chest. 1995;107:1532-37.

14. Melloni G, Carretta A, Ciriaco P, Negri G, Voci C, Augello G, et al. Decortication for chronic parapneumonic empyema: results of a prospective study. World J Surg. 2004;28:488-93.

15. Andrews NC, Parker EF, Shaw RR, Wilson NJ, Webb WR. Management of nontuberculous empyema. Am Rev Respir Dis. 1962;85:935-36.

16. Soriano T, Alegre J, Alemán C, Ruiz E, Vásquez A, Carrasco JL, et al. Factors influencing length of hospital stay in patients with bacterial pleural effusion. Respiration. 2005;72:587-93.

17. Light RW. Parapneumonic effusions and empyema. Proc Am Thorac Soc. 2006;3:75-80.

18. Thourani VH, Brady KM, Mansour K, Miller JI, Lee RB. Evaluation of treatment modalities for thoracic empyema: A cost-effective analysis. Ann Thorac Surg. 1998;66:1121-27.

19. Drain AJ, Ferguson JI, Sayeed R, Wilkinson S, Ritchie A. Definitive management of advanced empyema by two-window video-assisted surgery. Asian Cardiovasc Thorac Ann. 2007;15:238-39.

20. Kearney SE, Davies CW, Davies RJ, Gleeson FV. Computed tomography and ultrasound in parapneumonic effusion and empyema. Clinical Radiology. 2005;55:542-47.

21. Molnar TF. Current surgical treatment of thoracic empyema in adults. Eur J Cardiothorac Surg. 2007;32:422-30. 


\section{ORIGINAL ARTICLE}

\section{FIGURE ONE}

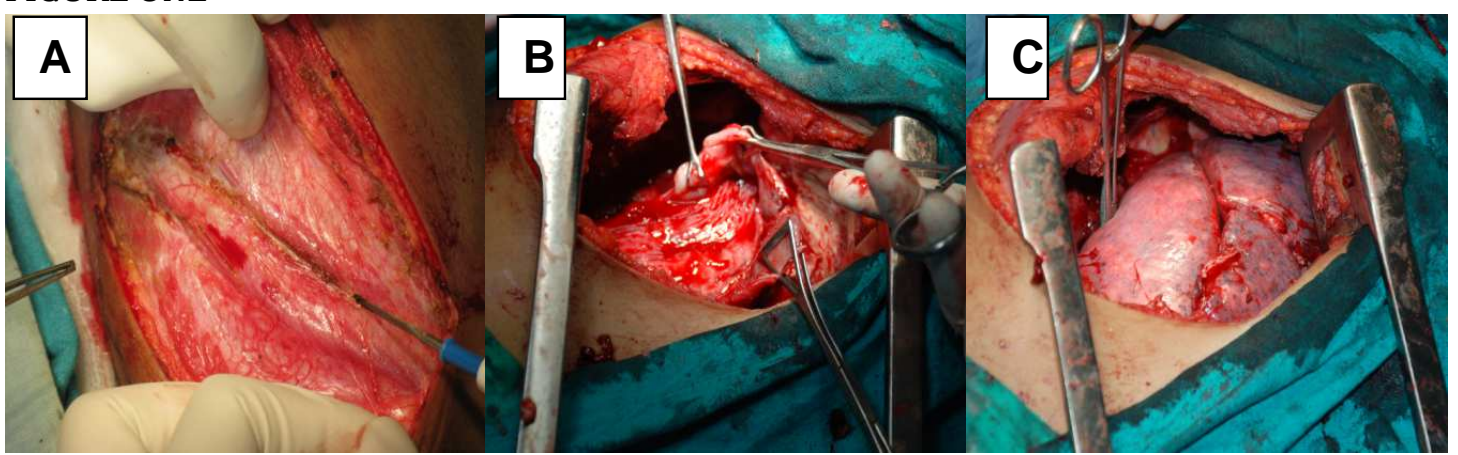

A. Chronic empyema. Note the rib crowding present B. Shows the thick peel present and the decortication being done. C. The lung has been completely decorticated and there is no residual dead space with complete lung expansion

\section{FIGURE TWO}

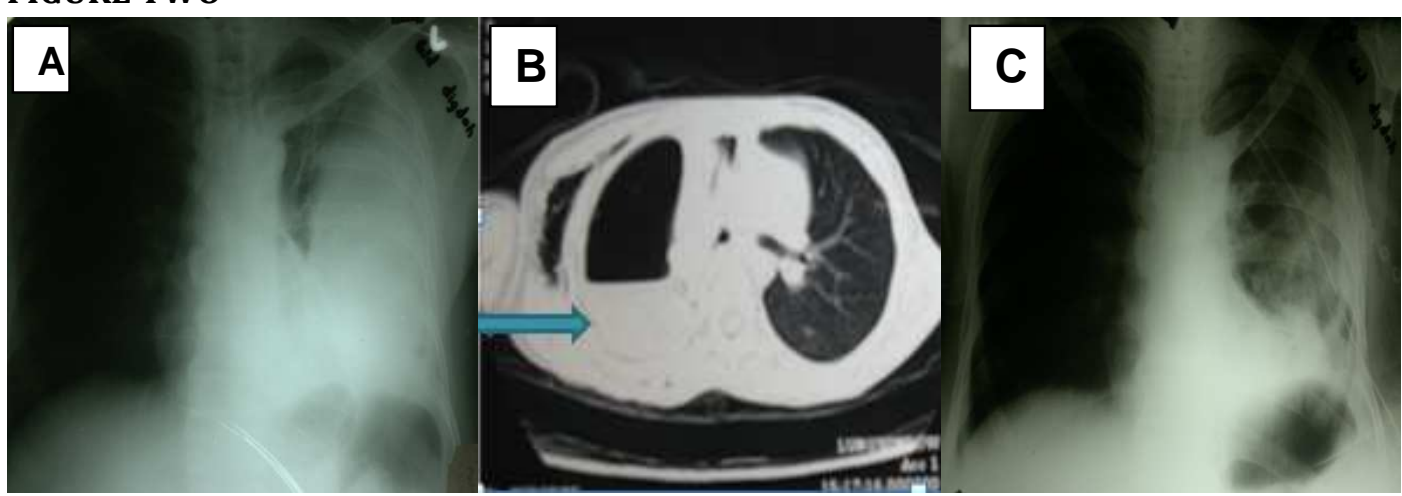

A. Chest roentgenogram shows a large left empyema. B. Computed tomography of the chest showing thick pleural cortex. C. Postoperative chest roentgenogram with excellent pulmonary expansion 